\title{
C-band ring cavity of virtual mirror BEFL with additional pump power
}

\begin{abstract}
A Multiwavelength BEFL with virtual mirror concept is demonstrated with an additional pump power to provide higher gain. $616 \mathrm{~mW}$ provided by a $975 \mathrm{~nm}$ pump is added to the conventional of $130 \mathrm{~mW} 1480 \mathrm{~nm}$ pump. Wide tuning range of $39 \mathrm{~nm}$ wavelengths was achieved and maximum of 9 laser lines was observed from the proposed design.
\end{abstract}

